\title{
The Detection of Low-Luminosity Carbon Stars in the Leo II and Fornax Dwarf Galaxies
}

\author{
GÉRARD MURATORIO $^{1}$ and MARC AZZOPARDI ${ }^{1,2}$ \\ 1 Observatoire de Marseille, Marseille, France \\ 2 Canada-France-Hawaii Telescope Corporation, Kamuela HI, U.S.A.
}

Our very deep low-resolution spectroscopic surveys of the Leo II and Fornax dwarf spheroidal galaxies resulted in the identification of several new faint carbon star candidates. For this purpose we used, in the slitless spectroscopic mode, various spectrographs attached to the ESO 4-m class telescopes and CFHT. All observations were carried out through an interference filter $\left(\lambda_{0}=\right.$ $4880 \AA ; \Delta \lambda \simeq 1000 \AA$ ) to narrow the instrumental spectral domain, thus keeping the number of overlapping images as low as possible. In this spectral region (4300-5300 $\AA$ ), carbon stars can be identified through their pronounced Swan $\mathrm{C}_{2}$ bands at 4737 and $5165 \AA$. A semi-automatic procedure in the MIDAS environment led us to select promising candidates whose carbon-star nature was then confirmed by medium-resolution slit spectroscopy. Data reduction techniques and results are shown. 